Revista de Psicología de la PUCP. Vol. XVII, 1, 1999.

\title{
PSICOPATOLOGÍA EN ADOLESCENTES DE LIMA SEGÚN EL INVENTARIO DE PROBLEMAS CONDUCTUALES DE ACHENBACH
}

\author{
Alegría Majluf' \\ Universidad Peruana Cayetano Heredia
}

El Inventario de Problemas Conductuales de Achenbach (confiabilidad tes/retest $=.92$ ) fue administrado a 908 adolescentes de Lima, cuyas edades estaban entre los 11 y 18 años. El grupo control (normal) estuvo conformado por 621 adolescentes y el grupo experimental (muestra clínica con padres divorciados) estuvo conformado por 287. Los resultados indican que el grupo experimental presentó puntajes significativamente superiores $(T \geq 70)$ frente al grupo control $(T<60)$, presentando el primer grupo de indicadores de patología. Más aún, en la mujeres se observó una tendencia no significativa a obtener puntajes $T$ superiores a los varones. Se concluye que el Inventario de Achenbach es un instrumento válido para la detección rápida de niños que presentan problemas de conducta y que requieren de una intervención psicológica o psiquiátrica, en el Perú.

Palabras claves: adolescencia, problemas de conducta, inventarios.

Adolescents psychopatology in Lima following the Achenbach Inventory of Behavioral Problems The Achenbach Inventory of Behavioral Problems (rest/retest reliability $=.92$ ) was administered to 908 adolescents in Lima, aged from 11 to 18 years, 621 coming from the control group (normal) and 287 from the experimental group (clinical group with divorced parents). Results showed that the experimental group exhibited significantly higher scores $(T \geq 70)$ than the control group $(T<60)$, the former reaching pathology level indicators. Moreover, a non-significant tendency to obtain higher $T$ scores is more observed in females than in males. From the study results we can state that the Achenbach Inventory is a valid instrument for a screening process of children exhibiting behavioral problems that require psychological or psychiactric intervention in Peru.

Key words: adolescence, hehavioral problems, inventories.

1. Es Profesora Principal de Psicología de la Universidad Particular Cayetano Heredia. Obtuvo el título de bachiller en Psicología en la Universidad Nacional Mayor de San Marcos. Realizó una maestría sobre desarrollo infantil en la Universidad Estatal de Iowa. Obtuvo el doctorado en el California School of Professional Psychology. Ha realizado estudios y publicaciones en el campo de la psicología del desarrollo y marginalidad, centrándose en la capacidad cognitiva y el léxico. Forma parte del Comité Editorial de la Revista de Psicología de la PUCP y es miembro de varias sociedades profesionales extranjeras. Dirección: José Quiñones 175, Lima 18. 

En el Perú, la investigación epidemiológica en relación a la psicopatología infantil y juvenil es incipiente. El primer estudio fue realizado por Mariátegui, Alva y De León en 1969 en Lince (distrito de Lima), con una muestra representativa de la zona. En la primera etapa del estudio se administró un Cuestionario para detectar casos con problemas los que posteriormente fueron entrevistados y diagnosticados por psiquiatras. En relación a la población infantil de 0 a 14 años se encontró una prevalencia de $13.2 \%$.

Dicho estudio, realizado hace 28 años, utilizó una metodología que es cuestionada actualmente. Asimismo, se observa que el sistema taxonómico en psicopatología de niños muestra muchas discrepancias. Cantwell y Carlson (1987) señalan que el sistema de clasificación no es correcto y está basado en conceptos teóricos y que deberían estar fundamentados en los hechos, ser fiable, válido, tener en cuenta el aspecto evolutivo y ser capaz de diferenciar categorías.

Varios investigadores insatisfechos con los métodos tradicionales han optado por los métodos empíricos. Entre estos se destaca Achenbach (1991) quien propone que la descripción y la clasificación de la psicopatología infantil debe enmarcarse dentro del contexto evolutivo del niño, ya que una conducta puede ser normal o no, dependiendo de la etapa evolutiva en que se encuentre; además, añade que la descripción de la conducta debe proveerse en formato estandarizado. Para ello, Achenbach (1991) elaboró Inventarios de Problemas Conductuales y Destrezas Sociales para niños entre 14 y 11 años y para jóvenes entre 11 y 18 años. Estos inventarios tenían por objeto comparar niños utilizando un criterio uniforme. Su objetivo fue proporcionar un instrumento estandarizado, útil para clínicos e investigadores al margen de su posición teórica. De esta manera, un lenguaje descriptivo facilitaría la discusión entre las diferentes orientaciones. 
El trabajo de Achenbach (1991) es un avance innovador que pretende configurar categorías diagnósticas en base a la forma como se agrupan los síntomas de los niños que presentan trastornos emocionales y conductuales valiéndose del análisis de componentes y del análisis factorial.

Achenbach (1991), no impone a priori creencias acerca de qué síntomas existen, mas bien éstas se derivan cualitativamente de pruebas estandarizadas acerca de problemas, calificados por los jóvenes en el inventario (Youth Self Report-YSR, 1991).

El puntaje total, el puntaje $T$ y los percentiles para cada una de las escalas permite comparar los puntajes logrados por un joven con aquellos obtenidos por una muestra normal. El punto de corte $(T=.70)$ permite distinguir entre jóvenes que presentan patología de aquellos normales y marginales.

El autor considera que el inventario de jóvenes puede ser muy útil si se administra en la primera entrevista en colegios y servicios de salud, pues los resultados pueden orientar la entrevista y ayudan al joven a hablar sobre sus problemas. Además, el inventario permite comparar la autopercepción del adolescente con los datos aportados por los padres o maestros en los inventarios elaborados para ellos.

Tanto los inventarios para nin̄os como para adolescentes han sido traducidos en más de 33 idiomas y administrados en numerosos servicios de salud, así como en investigaciones, brindando abundante evidencia e información teórica y empírica que respalda la aplicación del inventario (Achenbach, 1991; Achenbach y Brown, 1991).

En la traducción española Rubio-Stipec et al. (1990) se estudió la consistencia interna, así como la validez concurrente del inventario en Puerto Rico. En relación a la presencia o ausencia de diagnósticos mediante el DSM III, se encontró que los resultados concordaban con aquellos logrados con el inventario para niños. Es decir, el puntaje $T$ total de 70 o más indicaba probabilidad de ser diagnosticado como caso psiquiátrico 
Psicopatología en adolescentes de Lima según el inventario de problemas conductuales...

que necesita atención. Se concluyó que este inventario evidenciaba ser un excelente instrumento de detección de psicopatología, para fines epidemiológicos.

La traducción española ha sido aplicada en numerosos estudios. Entre estos, en la Universidad de Columbia en Chile por Montenegro (1983, 1987), y en Puerto Rico por Bird et al. (1987, 1988, 1990), evidenciándose su adecuación como instrumento para realizar estudios epidemiológicos.

En Lima, en un estudio reciente, Livia y Ortiz (1993) estimaron la prevalencia de psicopatología infantil en una población escolar de 6 a 11 años de una zona urbano-marginal de Collique-Lima. Se encontró un 18\% de patología, siendo más acentuada en las niñas.

El estudio de Livia y Ortiz no incluyó adolescentes por lo que se consideró importante y valioso utilizar el otro inventario desarrollado por Achenbach (Youth Self Report-YSR, 1991) para conocer la incidencia de psicopatología o trastornos de conducta de jóvenes de 11 a 18 años de edad en una muestra representativa de Lima. De manera que el objetivo del presente estudio incluyó en primer lugar, establecer la confiabilidad y validez del inventario:

- Determinar si las respuestas del inventario son significativamente diferentes en niños referidos a consulta de salud mental y en una muestra similar, pero que no ha consultado en los últimos 6 meses.

- Identificar los efectos de variables sociodemográficas como edad, sexo, nivel socioeconómico y estado civil de los padres sobre las respuestas del inventario.

- Determinar normas para el grupo de niños no referidos con el fin de poder utilizar el inventario para efectos clínicos y de investigación en la población peruana.

- Proporcionar a los especialistas en salud mental un instrumento de fácil aplicación y de bajo costo estandarizado en Lima para la detección de los niños que requieran atención psiquiátrica o psicológica. 


\section{Metodología}

\section{Participantes}

Se trabajó con 908 jóvenes de ambos sexos de 11 a 18 años de edad, de los cuales 621 correspondían al grupo normal (GN) y 287 al grupo clínico (GC). El GN constituyó una muestra representativa de Lima, seleccionado de colegios particulares (188) y estatales (433). El GC estuvo conformado por jóvenes que asistían a centros de salud mental de 135 institutos particulares y 152 estatales. Se omitieron jóvenes con retardo mental o con problemas de aprendizaje. El 89\% del GN y el 80\% del GC procedía de Lima, mientras el resto provenía de provincias.

Como se aprecia en el Cuadro 1, no hubo diferencias de proporciones según la edad entre el GN y el GC.

\section{Cuadro 1}

Diferencia de Proporciones Entre Tipos de Grupos Seguin la Edad

\begin{tabular}{|c|c|c|c|}
\hline \multirow{2}{*}{ Edad } & \multicolumn{2}{|c|}{ Típo de grupo } & \multirow{2}{*}{7} \\
\hline & $\begin{array}{l}\text { Normal } \\
(\%)\end{array}$ & $\begin{array}{c}\text { Clínico } \\
(\%)\end{array}$ & \\
\hline $11-13$ & 31.7 & 34.1 & -0.71 \\
\hline $14-16$ & 55.2 & 49.8 & -1.51 \\
\hline $17-18$ & 13.0 & 16.0 & -1.18 \\
\hline
\end{tabular}

Las diferencias de proporción entre los sexos si llega a ser significativa habiendo una mayor proporción de hombres en el GN y de mujeres en el GC (Cuadro 2). 
Psicopatología en adolescentes de Lima según el inventario de problemas conductuales...

Cuadro 2

Diferencia de Proporciones Entre GN y GC Según el Sexo

\begin{tabular}{|c|c|c|c|c|}
\hline \multirow{2}{*}{ Sexo } & \multicolumn{3}{|c|}{ Tipo de grupo } & \multirow[t]{2}{*}{$Z$} \\
\hline & Normal & & $\begin{array}{c}\text { Clínico } \\
\%\end{array}$ & \\
\hline Hombres & 60.2 & & 50.2 & $2.84^{*}$ \\
\hline Mujeres & 39.8 & - & 49.8 & $-2.84^{*}$ \\
\hline
\end{tabular}

${ }^{*} p<.01$

En cuanto al estado civil de los padres, la diferencia entre los grupos llega a ser significativa. En el GN hay una mayor proporción de padres que viven juntos. En el caso del GC existe mayor proporción de padres separados. Los detalles se muestran en el Cuadro 3.

Cuadro 3

Diferencia de Proporciones Entre Tipos de Muestra Según el Estado Civil de los Padres

\begin{tabular}{|lccc|}
\hline Estado civil & Típo de grupo & $Z$ \\
de los padres & Normal & Clínico & \\
\cline { 2 - 4 } & $\%$ & $\%$ & \\
\hline Viven juntos & 82.4 & 69.0 & $4.31^{*}$ \\
Separados & 11.0 & 21.6 & $-3.85^{*}$ \\
Divorciados & 2.6 & 2.4 & 0.18 \\
Muertos & 0.0 & 0.3 & -0.98 \\
Uno muerto & 4.0 & 5.6 & -1.01 \\
No especifica & 0.0 & 1.0 & -1.63 \\
\hline
\end{tabular}

${ }^{*} p<.01$ 


\section{Instrumentos}

Se administró el Inventario de Autoreporte a los 908 jóvenes (621 GN y 287 GC) de 11 a 18 años (Youth Self Report-YSR, 1991. Protocolo en Apéndice A). Se obtuvo del autor una traducción en español que se revisó para adecuarla a la población de Lima, sin realizar mayores cambios. Se administró sólo el Inventario de Problemas Conductuales y no el de Destrezas Sociales, pues éste no se adecuaba a la realidad peruana. Para la administración y calificación se siguió las indicaciones de Achenbach (Youth Self Report, 1991). El índice de confrabilidad del inventario a través del testretest (en el curso de siete días) fue de .92. La validez de la prueba se estableció mediante un criterio clínico externo ya que la prueba discrimina entre el grupo normal y clínico. Este último grupo obtuvo una proporción significativamente más alta de puntajes 70 a 80 (que indica patología), que el GN (Cuadro 4).

Debido a que el objetivo principal del estudio fue determinar baremos para efectos clínicos y de investigación y como instrumento screening, se construyeron normas únicas para el índice total de la muestra normal. La tabla de conversión de los puntajes brutos a puntajes $T$ se encuentra en el Apéndice B.

No se pudo derivar los síndromes del inventario debido a que la muestra clínica era muy pequeña para tal fin.

\section{Resultados}

Para comprobar las hipótesis de diferencias de medias y proporciones se utilizó la prueba $Z$. Uno de los supuestos para la aplicación de dicha prueba es la normalidad de la distribución de los puntajes, requisito que se cumple tanto en la muestra normal como en la clínica.

En cuanto a los resultados en el Cuadro 4 se advierte que en el rango de puntajes $T$ de 70 a 80 (patología) y en de 59 a 69 (marginal), la proporción de jóvenes del GC es significativamente más alta que el del 
Psicopatología en adolescentes de Lima según el inventario de problemas conductuales...

GN. Mientras que en el rango 48-58 (no patología), la proporción de jóvenes del GN es significativamente mayor que la del GC.

Cuadro 4

Diferencia de Proporciones Entre GN y GC Según el Puntaje $T$

\begin{tabular}{|c|c|c|c|}
\hline \multirow{2}{*}{ Puntaje T } & \multicolumn{2}{|c|}{ Tipo de grupo } & \multirow{2}{*}{$Z$} \\
\hline & $\begin{array}{l}\text { Normal } \\
\%\end{array}$ & Clinico & \\
\hline $26-36$ & 7.9 & 8.0 & -0.05 \\
\hline $37-47$ & 35.9 & 34.8 & 0.32 \\
\hline $48-58$ & 41.9 & 31.9 & $2.97^{* *}$ \\
\hline $59-69$ & 11.6 & 16.7 & $-2.00^{*}$ \\
\hline $70-80$ & 2.6 & 9.1 & $-3.54^{* *}$ \\
\hline $81-90$ & 0.2 & 0.3 & -0.28 \\
\hline
\end{tabular}

${ }^{*} p<.05{ }^{* *} p<.01$

A partir de los 17-18 años, el puntaje $T$ obtenido por la GC es significativamente más alto que la de la GN, tal como se puede apreciar en el Cuadro 5.

Cuadro 5

Diferencia de Medias de los Puntajes T entre GN y GC Según la Edad

\begin{tabular}{|c|c|c|c|}
\hline \multirow{2}{*}{ Edad } & \multicolumn{2}{|c|}{ Tipo de grupo } & \multirow{2}{*}{$Z$} \\
\hline & $\begin{array}{c}\text { Normal } \\
\%\end{array}$ & Clínico & \\
\hline $11-13$ & 47.49 & 48.95 & -1.05 \\
\hline $14-16$ & 50.10 & 50.28 & -0.18 \\
\hline $17-18$ & 49.72 & 59.38 & $-5.32^{*}$ \\
\hline
\end{tabular}

${ }^{*} p<.01$ 
La diferencia entre las medias de los puntajes $T$ es significativamente mayor en el GC cuando los padres vivían juntos o estaban divorciados (Cuadro 6).

\section{Cuadro 6}

Diferencia de Medias de los Puntajes Tentre el GN y el GC Según el Estado Civil de los Padres

\begin{tabular}{|lccl|}
\hline $\begin{array}{l}\text { Estado civil } \\
\text { de los padres }\end{array}$ & Tipo de grupo & $Z$ \\
& $\begin{array}{c}\text { Normal } \\
\%\end{array}$ & $\begin{array}{c}\text { Cĺ́nico } \\
\%\end{array}$ & \\
\hline Viven juntos & 48.62 & 50.54 & $-2.15^{*}$ \\
Separados & 52.60 & 51.76 & -0.45 \\
Divorciados & 51.97 & 63.52 & $-2.40^{*}$ \\
Muertos & 0.00 & 71.19 & - \\
\hline
\end{tabular}

${ }^{*} p<.05$

En cuanto a la diferencia de las medias entre los sexos, el GN fue significativa en el rango de los puntajes $T$ 26-36 (no patología), en que los varones obtuvieron una proporción más alta $(10 \%)$ en relación a las mujeres (4.5\%). En el GC, en el rango de 37-47, los varones presentaron también una proporción más alta $(46.7 \%)$ que las mujeres (28\%). En el rango 70-80 (patología) las mujeres mostraron una proporción más alta $(11.9 \%)$ que los varones $(6.3 \%)$, aunque la diferencia no fue significativa.

Respecto a la diferencia entre los puntajes $T$ entre los estratos socioeconómicos -estimada según la asistencia a colegio particular o estatal, tanto de los jóvenes del GN como del GC- resultó significativa sólo entre los jóvenes de ambos grupos que asistían a colegios estatales. Como se puede apreciar en el Cuadro 7, los jóvenes del GC lograron una media significativa más alta, en comparación con los del GN. 
Psicopatología en adolescentes de Lima según el inventario de problemas conductuales...

Cuadro 7

Diferencia de medias de jóvenes del GN y del GC de Colegios Estatales

\begin{tabular}{|ccc|}
\hline \multirow{2}{*}{$\bar{X}$ colegio estatal } & \\
\cline { 2 - 3 } & Normal & $Z$ \\
\hline 58 & 63 & $2.38^{*}$ \\
\hline
\end{tabular}

${ }^{*} p<.05$

\section{Discusión}

Los resultados del estudio realizado con una población escolar de jóvenes de ambos sexos de 11 a 18 años, representativa de Lima, confirma la validez del inventario (YSR) como un instrumento para la detección gruesa de probable psicopatología. En este sentido, se han reportado hallazgos similares por Montenegro (1987) en Chile, Bird et al. (1987, 1988, 1990) en Puerto Rico, así como en múltiples estudios de varios países referidos por Achenbach (1991) y Achenbach y Brown (1991).

Los jóvenes con altos índices de problemas conductuales, con puntajes $T$ de 70 o más constituyen un 2\% del GN y un 9\% del GC. Esto coincide con los estudios de Montenegro (1987). Es probable que de haber existido problemas más específicos mediante los síndromes, no se hayan podido detectar, por no haber contado con un GC mayor. En este sentido, tal vez el porcentaje se hubiera elevado de 15 a $18 \%$, resultado obtenido por Livia con niños de 6 a 11 años en un estudio realizado en Collique-Lima.

En cuanto a las limitaciones del inventario se advierte su reducida capacidad para detectar la gravedad de la psicopatología debido a que si bien los puntajes cuantitativos permiten una buena discriminación, no siempre un mayor porcentaje va a significar una patología más grave, por lo que se considera valiosa la sugerencia de Achenbach de contrastar los datos aportados por el joven, que reflejen su autopercepción, con los datos consignados por los padres y/o maestros en sus inventarios respectivos. 
Achenbach considera que un profesional responsable debería integrar todos los datos y si fuera necesario realizar exámenes adicionales para lograr una visión más completa de la problemática del joven y orientar la ayuda que necesita.

Por último, es importante señalar como lo indicara Achenbach (1991), que el inventario (YSR) sería sumamente valioso como instrumento para estudios transculturales. El autor considera que el instrumento debidamente calibrado en diferentes países y culturas permitiría el avance del estudio de la psicopatología.

Se sugiere replicar el estudio y ampliar la muestra clínica para poder establecer los síndromes en la población de jóvenes limeños.

\section{Referencias}

Achenbach, T. y Edelbrock, C. (1981). Behavioral problems and competence reported by parents of normal and distorbed children aged four to sixteen. Monographs of Society for Research in Child Development, 46 (1 serial 188).

Achenbach, T. (1991). Manual for the Youth Self Report and profile. Burlington VT: University of Vermont.

Achenbach, T., Howell, C., Quay, H. y Conners, C. (1991). National survey of problems and competencies among four to eighteen years old. Monographs of the Society of Research in Child Development, 56(3) (Serial 225).

Achenbach, T. y Brown, J. (1991). Bibliography of published studies using the Child Behavior Checklist and related Materials Burlington. Vermont: Universidad de Vermont.

Bird, H., Canino, G., Gould, M., Ribera, J., Rubio-Stipec, M., Woodburry, M., Huerta, S. y Sesman, M. (1987). Use of Child Behavior Checklist as a screening instrument for epidemilogical research inc child psychiatry. Results of the pilot study. Journal of the American Academy of Child and Adolescent Psychiatry, 26(2). 
Psicopatología en adolescentes de Lima según el inventario de problemas conductuales...

Bird, H. y Canino, G. (1988). Estimates of the prevalence of childhood maladjustment in community survey in Puerto Rico. Archives of General Psychiatry, 45(2).

Bird, H., Rubio-Stipec, M., Canino, M. y Gould, M. (1990). The internal consistency and concurrent validity of a spanish translation of the Child Behavior Checklist. Journal of Abnormal Child Psychology, 18(4), 293-406.

Cantwell, G. y Carlson, G. (1987). Transtornos afectivos en la infancia y la adolescencia. Barcelona: Martinez Roca.

Livia, Y. y Ortiz, M. (1993). Inventario de Problemas Conductuales y Destrezas Sociales de Achenbach. Lima: Margen.

Mariátegui, J., Alva, V. y De León, O. (1969). Epidemiología psiquiátrica de un distrito urbano de Lima. Revista de Neuropsiquiatría, 115(2).

Montenegro, H., Braile, S., Edwards, M., Izquierdo, T. y Malter, S. (1983). Salud mental del escolar. Estandarización del Inventario de Problemas Conductuales y de Destrezas Sociales de Achenbach en niños de 6 a 11 años. Santiago de Chile: CIDE.

Montenegro, H., Braile, S. y Seguel, J. (1987). Prevalencia de transtornos psíquicos en la población escolar de Santiago de Chile. Acta Psiquiátrica Psicológica. América Latina, 22, 316-325. 


\section{Apéndice A 1}

Estamos haciendo un estudio para conocer algunas características psicológicas y sociales de los JÓVENES DE LA CIUDAD DE LIMA.

Te pedimos contestar con la mayor sinceridad a todas las preguntas que te hacemos en este cuestionario.

No hay respuestas correctas ni incorrectas y no es necesario que escribas tu nombre.

La información que nos proporciones será muy valiosa para el éxito de este estudioy para poder ayudar a los jóvenes que lo necesitan.

I. DATOS PERSONALES:

1. ¿Cuántos años tienes?

2. Sexo: $M$ $\mathrm{F}$

3. ¿En qué departamento naciste?

4. Tus padres (marca sólo una respuesta)

a. Viven juntos

b. Están separados

c. Están divorciados

d. Los dos han muerto

e. Uno de ellos ha muerto

II. ESCOLARIDAD

1. ¿Asistes al colegio? SI NO

2. ¿En qué año estás?
a. Primero
b. Segundo
c. Tercero 
Psicopatología en adolescentes de Lima según el inventario de problemas conductuales...
d. Cuarto
( )
e. Quinto
( )

3. Tu colegio es:
a. Estatal
( )
b. Particular
( )
c. Parroquial
( )
d. Otro
( )
Especifica

5. ¿Cómo se llama tu colegio? 


\section{LISTA DE CHEQUEO DE PROBLEMAS CONDUCTUALES Y SENTIMIENTOS}

Instrucciones: A continuación se presentan algunas oraciones que describen la conducta de algunos muchachos (as). Lee cada una atentamente y responde con sinceridad si en cualquier época, ahora o durante los seis últimos meses te ha ocurrido a ti lo mismo. Marca con un aspa sólo una respuesta para cada oración bajo la columna correspondiente.

1. Actúo como si fuera menor de lo que soy

$\begin{array}{cccc}\text { Nunca } & \begin{array}{c}\text { Algunas } \\ \text { veces }\end{array} & \begin{array}{c}\text { Casi } \\ \text { siempre }\end{array} \\ 0 & 1 & 2 & - \\ 0 & 1 & 2 & - \\ 0 & 1 & 2 & - \\ 0 & 1 & 2 & - \\ 0 & 1 & 2 & - \\ 0 & 1 & 2 & - \\ & & & \\ 0 & 1 & 2 & -\end{array}$

10. No puedo estar quieto(a) en mi asiento, soy inquieto(a) e hiperactivo(a)

11. Soy demasiado dependiente, apegado a los adultos.

12. Me siento solo(a)

13. Me siento confuso, como en las nubes

14. Lloro mucho

15. Soy bastante honesto, sincero (a)

16. Soy malo con los demás

17. Sueño despierto

18. he tratado de hacerme daño a mi mismo

19. Trato de que me presten atención

20. Destruyo mis cosas

21. Destruyo las cosas de otras personas

22. Desobedezco a mis padres

$$
\begin{gathered}
\text { Ahora o durante los últimos } \\
\text { seis meses }
\end{gathered}
$$

2. Padezco alergias

3. Discuto mucho

4. Padezco asma

5. Me comporto como si fuera del sexo opuesto 
Psicopatología en adolescentes de Lima según el inventario de problemas conductuales...

Ahora o durante los últimos

seis meses

Nunca Algunas Casi

veces siempre

23. Desobedezco en la escuela

24. No como tan bien como debería

25. No me llevo bien con otros muchachos

26. No me siento culpable después de hacer algo que no debería

27. Me siento celoso (a) de los demás

28. Estoy dispuesto a ayudar a los demás cuando necesitan ayuda

29. Le tengo miedo a ciertos animales, situaciones o lugares (no incluye la escuela).

Escribe:

30. Me da miedo ir a la escuela

31. Tengo miedo de pensar o hacer algo malo

32. Siento que tengo que ser perfecto

33. Siento que nadie me quiere

34. Siento que los demás me quieren perjudicar o fastidiar

35. Me siento inferior, que no valgo nada

36. Me golpeo a menudo accidentalmente

37. Me meto en muchas peleas

38. Los otros a menudo se burlan de mí

39. Me junto con muchachos(as) que se meten en problemas

40. Oigo cosas que nadie parece oir.

Escribir:

41. Hago las cosas sin pensar

42. Me gusta estar solo(a)

43. Digo mentiras o hago trampas

44. Me como las uñas

45. Soy nervioso o tenso

46. Tengo movimientos nerviosos, tics. Escribe:

47. Me dan pesadillas

48. No le caigo bien a otros muchachos (as)

49. Puedo hacer ciertas cosas mejor que otros

50. Soy demasiado ansioso (a) o tengo mucho miedo 0

51. Me siento mareado
0

0

0

0

0

0

$\begin{array}{lll}1 & 2 & - \\ 1 & 2 & - \\ 1 & 2 & -\end{array}$

0

0

0

0

0

0

0

0

0

0

0

0

0

0

0

0

0

0

0

0

0
0 
52. Me siento demasiado culpable

53. Como demasiado

54. Me siento demasiado cansado

55. Estoy por encima del peso normal

56. Problemas físicos sin causa médica conocida

\begin{tabular}{cccc} 
Ahora o durante los últimos \\
seis meses \\
Nunca & Algunas & Casi \\
\multicolumn{5}{c}{ veces } & siempre \\
0 & 1 & 2 & - \\
0 & 1 & 2 & - \\
0 & 1 & 2 & - \\
0 & 1 & 2 & - \\
0 & 1 & 2 & - \\
0 & 1 & 2 & - \\
0 & 1 & 2 & - \\
0 & 1 & 2 & - \\
& & & \\
0 & 1 & 2 & - \\
0 & 1 & 2 & - \\
0 & 1 & 2 & - \\
0 & 1 & 2 & - \\
& & & \\
0 & 1 & 2 & - \\
0 & 1 & 2 & -
\end{tabular}

57. Ataco físicamente a otras personas

a. Dolores de cuerpo

b. Dolores de cabeza

c. Ganas de vomitar, náuseas, mareos

d. Problemas con los ojos.

Describe

e. Picazón o erupciones en la piel

f. Dolores de barriga

g. Vómitos

h. Otros

Describe:

58. Me meto los dedos a la nariz, me raspo con las uñas, la piel u otras partes del cuerpo

59. Puedo ser bastante amistoso y me gusta la gente

60. Me gusta probar cosas nuevas

61. Mi trabajo escolar es deficiente

62. Soy un tanto torpe, poco coordinado

63. Prefiero estar con muchachos (as) mayores que yo 0

64. Prefiero estar con muchachos (as) menores que yo 0

65. Me niego a hablar

66. Repito ciertas acciones una y otra vez

67. Me he fugado de mi casa

68. Grito mucho

69. Soy reservado (a), mantengo mis cosas en secreto 0

70. Veo cosas que la otra gente parece no ver.

Describe:

71. Estoy demasiado pendiente de mí mismo, siento vergüenza con facilidad

72. Prendo fuego

73. Tengo habilidad para hacer cosas con las manos 0

74. Me gusta hacerme el (la) gracioso (a)

o el payaso (a)

0

0

0

64 
Psicopatología en adolescentes de Lima según el inventario de problemas conductuales...

75. Soy tímido(a)

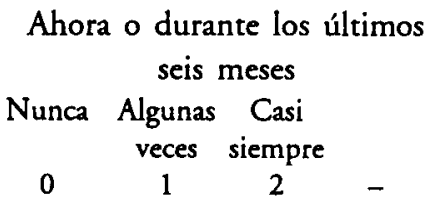

76. Duermo menos que la mayoría de los muchachos (as)

77. Duermo más que la mayoría de los muchachos (as), durante el día y/o la noche

78. Tengo buena imaginación

79. Tengo problemas con el habla.

Describe

80. Hago valer mis derechos

81. Me apodero de cosas de mi casa que no me pertenecen

82. Me apodero de cosas que no son de mi casa

83. Almaceno o guardo cosas que no necesito

84. Hago cosas que las demás personas consideran raras

85. Pienso cosas que las demás personas considerarían raras.

Describe

86. Soy porfiado

87. Mi estado de ánimo o mis sentimientos cambian a cada momento

88. Disfruto al estar con otras personas

0

0

0

0

0

0

0

0

0

1

2

0

0

$\begin{array}{lll}1 & 2 & - \\ 1 & 2 & -\end{array}$

89. Soy desconfiado, receloso

90. Maldigo o digo malas palabras

91. Pienso en matarme

92. Me gusta hacer reir a los demás

93. Hablo demasiado

94. Fastidio a menudo a los demás

95. Tengo mal genio

96. Me preocupo demasiado por cosas sexuales

97. Amenazo a otros con que les voy a hacer daño

98. Me gusta ayudar a los demás

99. Me preocupo demasiado por la limpieza $y$ por el orden

100. Tengo dificultad en dormir Describe:

101. Falto a la escuela 
102. No tengo mucha energía

103. Me siento infelíz, triste, deprimido

104. Soy más ruidoso "bocón" que otros muchachos
Ahora o durante los últimos seis meses

105. Tomo alcohol, fumo tabaco o uso drogas (no incluyas medicinas bajo receta médica).

Describe:

106. Trato de ser justo

107. Disfruto de un buen chiste

108. Me gusta tomar la vida alegremente

109. Trato de ayudar a los demás

110. Quisiera ser del sexo opuesto

111. Trato de no relacionarme con los demás

112. Me preocupo demasiado

Nunca Algunas Casi
veces siempre

113. Dime cualquier cosa que describa tus sentimiento

$\begin{array}{lll}1 & 2 & - \\ 1 & 2 & - \\ 1 & 2 & -\end{array}$

0 , tu comportamiento o tus intereses: 
Psicopatología en adolescentes de Lima según el inventario de problemas conductuales...

\section{INVENTARIO DE PROBLEMAS CONDUCTUALES DE ACHENBACH}

\section{Manual de Calificación}

\section{Aspectos generales}

1. No se califica el Inventario si se han omitido o no se han respondido 8 ítems, excluyendo los siguientes: 2,4 y $56 \mathrm{~h}$.

2. Si la persona marca 2 opciones para el mismo ítem considere sólo el puntaje como 1.

3. Observe que sólo hay 103 ítems de problemas a pesar que los ítems fluctúan de 1 al 112.

4. Los ítems 2 y 4 no se cuentan en el puntaje total de problemas.

5. Hay 8 ítems del 56 a al $56 \mathrm{~h}$.

6. Los 16 ítems que son socialmente deseables se omiten del puntaje total; éstos son los siguientes: $6,15,28,49,59,60,73,78,80,88$, 92, 98, 106, 107, 108 у 109.

7. Los comentarios que realizan los jóvenes al finalizar la prueba (ítem 113) no son calificados.

\section{Calificación de los items}

1. El puntaje que el joven haya marcado debe colocarse al costado de cada ítem, en la línea al final del ítem.

2. Los comentarios escritos por los jóvenes en relación a cada uno de los ítems deben ser calificados considerando las siguientes pautas:

- Para cada problema señalado por el joven se califica sólo aquel que describe de forma más específica el problema porque puede ocurrir que ese mismo problema ya haya sido señalado en otro ítem.

- Para conductas extremas como prender fuego o intento de suicidio, si el joven señala que fue sólo una vez o marca " 0 " (cero) o no contesta, se le otorga el puntaje 1 . 
- Para las pruebas en las cuales el joven señala que "acostumbraba hacerlo", calificar de acuerdo a lo que el joven señala; a no ser que haya ocurrido antes de los seis meses especificados en las instrucciones.

- Cuando hay duda, calificar el ítem de acuerdo a lo que el joven señala, excepto en los siguientes ítems:

ftem 9: No puedo sacar de la cabeza algunos pensamientos. Este ítem no está restringido a las obsesiones. Puede incluir casi cualquier cosa, exceptuando los problemas que han sido señalados específicamente. Por ejemplo, si el joven escribe "sexo", sería más apropiado calificarlo en el ítem 96 Pienso demasiado en el sexo. Si lo que el joven indica no está considerado por otro ítem, entonces debe ser calificado de acuerdo a lo que el joven señala.

Ítem 40: Oigo cosas que nadie parece oir e ítem 70: Veo cosas que la otra gente no parece ver. Se refiere a experiencias como zumbidos o el ver manchas delante de los ojos. Se debe calificar, según lo que el joven ha señalado. No se califican experiencias que suceden bajo la influencia de las drogas o del alcohol.

ftem 46: Tengo movimientos nerviosos, tics. Si el joven señala que no puede estar sentado tranquilo, calificar sólo el ítem 10 .

Item 56d: Problemas con los ojos. No se califica el uso de anteojos, la miopía o cualquier problema visual de base orgánica.

Item 66: Repito ciertas acciones una y otra vez. Este ítem no está restringido sólo a compulsiones. Puede incluir casi cualquier cosa que el joven señale, exceptuando problemas específicos señalados en otros lugares o ítems. Por ejemplo, la tartamudez sería calificada de manera más apropiada en el ítem 79. 
Psicopatología en adolescentes de Lima según el inventario de problemas conductuales...

Ítem 77: Duerme más que la mayoria de los muchachos, durante el día y/o la noche. No se califica el deseo de estar en cama, pero sí las dificultades al levantarse.

Ítem 84: Hago cosas que las demás personas consideran raras e ítem 85: Pienso cosas que las demás personas considerarian raras. Si lo que el joven describe ha sido cubierto en forma más específica en otro ítem, calificar éste.

Ítem 105: Tomo alcohol, fumo tabaco o uso drogas (no incluye medicinas bajo receta médica). No se califica tabaco o medicación.

\section{Puntaje Total}

Para obtener el Puntaje Bruto Total, sume los puntajes "1" y "2" de cada uno de los ítems. Este puntaje se puede convertir en puntaje $T$, valiéndose del Apéndice 2, en el que se indica a la vez el percentil correspondiente. 


\section{Apéndice B}

Conversión del Índice de Problemas Conductuales a Puntajes $T$ y Percentiles

\begin{tabular}{|c|c|c|c|c|c|c|c|c|}
\hline Pre: & $\begin{array}{l}\text { Ptje. } \\
\text { Trans } \\
\text { formado }\end{array}$ & Perc. & $\begin{array}{l}\text { Prje. } \\
\text { Bruto }\end{array}$ & $\begin{array}{l}\text { Prje. } \\
\text { Trans } \\
\text { formado }\end{array}$ & Perc. & Prije, & $\begin{array}{l}\text { Prje } \\
\text { Trans } \\
\text { formado }\end{array}$ & Perc. \\
\hline 137 & 100 & \multirow[t]{22}{*}{100} & 89 & 81 & & 41 & 46 & \\
\hline 136 & 100 & & 88 & 80 & 94 & 40 & 44 & \\
\hline 135 & 99 & & 87 & 80 & 93 & 39 & 43 & \\
\hline 134 & 99 & & 86 & 79 & 92 & 38 & 42 & \\
\hline 133 & 98 & & 85 & 79 & & 37 & 41 & \\
\hline 132 & 98 & & 84 & 79 & & 36 & 40 & \\
\hline 131 & 98 & & 83 & 78 & 91 & 35 & 39 & \\
\hline 130 & 97 & & 82 & 78 & 90 & 34 & 38 & \\
\hline 129 & 97 & & 81 & 77 & & 33 & 37 & \\
\hline 128 & 96 & & 80 & 77 & 88 & 32 & 36 & 7 \\
\hline 127 & 96 & & 79 & 76 & 87 & 31 & 34 & \\
\hline 126 & 96 & & 78 & 76 & 86 & 30 & 33 & 6 \\
\hline 125 & 95 & & 77 & 76 & & 29 & 32 & 5 \\
\hline 124 & 95 & & 76 & 75 & & 28 & 31 & 4 \\
\hline 123 & 94 & & 75 & 75 & & 27 & 30 & \\
\hline 122 & 94 & & 74 & 74 & & 26 & 29 & \\
\hline 121 & 94 & & 73 & 74 & & 25 & 28 & 3 \\
\hline 120 & 94 & & 72 & 74 & & 24 & 27 & \\
\hline 119 & 93 & & 71 & 73 & & 23 & 26 & 2 \\
\hline 118 & 93 & & 70 & 73 & & 22 & 24 & \\
\hline 117 & 92 & & 69 & 72 & & 21 & 23 & \\
\hline 116 & 92 & & 68 & 72 & & 20 & 22 & 1 \\
\hline 115 & 91 & \multirow[t]{8}{*}{99} & 67 & 72 & & 19 & 21 & \\
\hline 114 & 91 & & 66 & 71 & & 18 & 20 & \\
\hline 113 & 91 & & 65 & 71 & & 17 & 19 & \\
\hline 112 & 90 & & 64 & 70 & & 16 & 18 & \\
\hline 111 & 90 & & 63 & 70 & & 15 & 17 & \\
\hline 110 & 89 & & 62 & 69 & & 14 & 16 & \\
\hline 109 & 89 & & 61 & 68 & & 13 & 14 & \\
\hline 108 & 89 & & 60 & 67 & & 12 & 13 & \\
\hline 107 & 88 & \multirow[t]{2}{*}{98} & 59 & 66 & & 11 & 12 & \\
\hline 106 & 88 & & 58 & 64 & & 10 & 11 & \\
\hline
\end{tabular}


Psicopatología en adolescentes de Lima según el inventario de problemas conductuales...

\begin{tabular}{|c|c|c|c|c|c|c|c|c|}
\hline $\begin{array}{l}\text { Ptie. } \\
\text { Bruto }\end{array}$ & $\begin{array}{l}\text { Ptie. } \\
\text { Trans } \\
\text { formado }\end{array}$ & Pere. & $\begin{array}{l}\text { Ptje: } \\
\text { Bruto }\end{array}$ & $\begin{array}{l}\text { Ptre. } \\
\text { Trans } \\
\text { formado }\end{array}$ & Perc. & $\begin{array}{l}\text { Ptje. } \\
\text { Bruto }\end{array}$ & $\begin{array}{l}\text { Ptje: } \\
\text { Trans } \\
\text { formado }\end{array}$ & Perc \\
\hline 105 & 87 & & 57 & 63 & & 9 & 10 & \\
\hline 104 & 87 & & 56 & 62 & & 8 & 9 & \\
\hline 103 & 87 & & 55 & 61 & & 7 & 8 & \\
\hline 102 & 86 & & 54 & 60 & & 6 & 7 & \\
\hline 101 & 86 & & 53 & 59 & & 5 & 6 & \\
\hline 100 & 85 & 97 & 52 & 58 & & 4 & 4 & \\
\hline 99 & 85 & & 51 & 57 & & 3 & 3 & \\
\hline 98 & 84 & & 50 & 56 & & 2 & 2 & \\
\hline 97 & 84 & & 49 & 54 & & 1 & 1 & \\
\hline 96 & 83 & & 48 & 53 & & 0 & 0 & \\
\hline 95 & 83 & & 47 & 52 & & & & \\
\hline 94 & 83 & 96 & 46 & 51 & & & & \\
\hline 93 & 82 & & 45 & 50 & & & & \\
\hline 92 & 82 & & 44 & 49 & & & & \\
\hline 91 & 81 & 95 & 43 & 48 & & & & \\
\hline 90 & 81 & & 42 & 47 & & & & \\
\hline
\end{tabular}

